\title{
Optimized power allocation strategies in hybrid optical satellite networks
}

Theodore Kapsis, Athanasios Panagopoulos

Theodore T. Kapsis, Athanasios D. Panagopoulos, "Optimized power allocation strategies in hybrid optical satellite networks," Proc. SPIE 11852, International Conference on Space Optics - ICSO 2020, 118525P (11 June 2021); doi: 10.1117/12.2600009

SPIE Event: International Conference on Space Optics - ICSO 2021, 2021, Online Only 


\section{International Conference on Space Optics-ICSO 2020}

Virtual Conference

30 March-2 April 2021

Edited by Bruno Cugny, Zoran Sodnik, and Nikos Karafolas
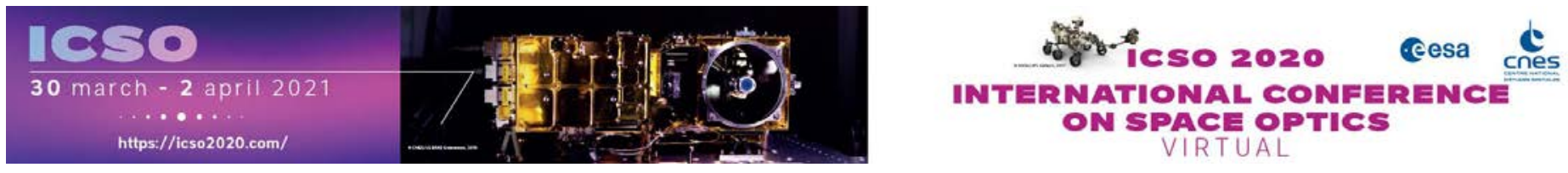

\section{Optimized power allocation strategies in hybrid optical satellite networks}

\section{Cesa issopoceatings lecnes}




\title{
Optimized Power Allocation Strategies in Hybrid Optical Satellite Networks
}

\author{
Theodore T. Kapsis ${ }^{\mathrm{a}}$, Athanasios D. Panagopoulos*a

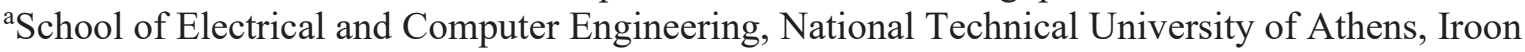 \\ Polytechniou 9, Athens-Zografou, GR15780, Greece.
}

\begin{abstract}
The globally introduced $5 \mathrm{G}$ mobile networks enable a variety of broadband applications from massive machine type communications (mMTC) to ultra-reliable low latency communications (URLLC). Within this context, optical technologies such as radio over fiber (RoF), radio over free space optics (RoFSO) and optical satellite networks are designated to provide excellent backhaul services. In this paper, the power optimization problem is investigated for a fully optical hybrid satellite network. Specifically, the dual hop decode-and-forward optical downlink is considered with a geostationary satellite source, an optical ground gateway and an optical user equipment. The power allocation problem is formulated as a convex optimization problem under separate total and peak power constraints and then a methodology is proposed for the maximization of system's capacity. Moreover, the proposed methodology takes into account the atmospheric attenuation, the optical channel correlation and turbulence effects and its performance is evaluated through numerical simulations and comparisons with other power allocation implementations. Results regarding the spectral efficiency are presented and commented proving the proposed methodology's superiority.
\end{abstract}

Keywords: Optical hybrid network, dual hop relay satellite, spatial multiplexing MIMO, optimum power allocation, atmospheric turbulence, scintillation, channel correlation, channel capacity.

\section{INTRODUCTION}

The Free Space Optics (FSO) technology is considered a mature type of wireless communication similar to Fiber Optics and complementary to RF radio-communications. The incorporation and deployment of FSO backhaul links in the $5 \mathrm{G}$ framework will be beneficial as they provide inexpensive installation costs, limited power consumption, low mass, enormous spectrum $(\mathrm{THz})$ as well as enhanced secrecy due to the directivity properties of the laser beams [1]-[2]. Optical satellite communication systems have been tested and their feasibility has already been demonstrated through various experiments with geostationary (GEO) satellites [3], low earth orbit (LEO) satellites [2], medium earth orbit (MEO) satellites [4]-[5], deep space satellites, nanosatellites among others. Despite the phenomenal characteristics of bidirectional optical satellite links, their availability is seriously degraded in the presence of foggy or cloudy weather due to the intense absorption and scattering [6]. However, the laser beam propagation is heavily dependent on the atmospheric turbulence even in cloud-free environments which inflicts beam spreading and mainly the so-called scintillation effects [7]. Scintillation is a temporal phenomenon causing received irradiance fluctuations because of the changes in the refractive index along the slant path [7].

The multiple input multiple output (MIMO) antenna systems and their usages e.g. multi-user MIMO, massive MIMO, distributed MIMO, V-BLAST are a core component in many wireless network standards as they offer beam-steering possibilities, higher data throughput via spatial multiplexing and advanced diversity techniques [8]. MIMO-based relay systems are commonly employed to improve reliability and coverage especially when the direct link qualities are poor and the need for cooperative or multi-hop communication emerges [9]. The relay transmission schemes are classified primarily into decode-and-forward (DF) and amplify-and-forward (AF) where the DF is better opted for good sourcerelay channels [9]. The power allocation problem for two-hop, MIMO-based relay networks has been studied in [10][11] and methodologies have been proposed for uncorrelated Rayleigh channels. Additionally, capacity bounds and outage analysis for all-optical relay-assisted log-normal links have also been reported in [12].

In this contribution, a power allocation methodology is proposed for a hybrid fully optical satellite network comprising of a GEO satellite multi-channel source, an optical ground relay station (gateway) and an optical user equipment (edge node). A dual hop, DF scheme is employed for the downlink optical channels that are affected by attenuation, 
scintillation and spatial correlation. The transmitting nodes have separate total available powers and peak powers therefore the power allocation optimization is performed with respect to both source's and relay's power constraints. The proposed methodology is based on the convexity theory described in [13]-[14] and the waterfilling algorithm [15] but also encompasses the statistical properties of the channel besides the optical channel state. Finally, numerical data are generated to simulate the examined system model for various channel settings i.e. scintillation profiles, correlation coefficients, and the methodology's performance is compared to other power distribution implementations to show the achieved spectral efficiency gain.

The remainder of the paper is structured as follows: In Section 2, the network architecture framework is given along with the hybrid optical MIMO technical characteristics. The major communication assumptions are also reported. In Section 3 , the systemic equations and important metrics are given and a power allocation methodology is proposed for the optimization of the system's capacity. In Section 4, numerical data are generated and simulations are executed. The numerical results are then presented followed by related remarks. Finally, Section 5 concludes this paper.

\section{NETWORK ARCHITECTURE FRAMEWORK}

The system under investigation is a three-node network consisting of a GEO satellite source equipped with $\mathrm{N}_{\mathrm{T}}$ optical transmit telescopes, an optical ground relay station (OGRS) with $\mathrm{N}_{\mathrm{T}}$ optical transmit and $\mathrm{N}_{\mathrm{R}}$ optical receive telescopes installed, and the optical ground user station (OGUS) with also $\mathrm{N}_{\mathrm{R}}$ optical receivers. The GEO-OGRS and OGRS-OGUS links form $\mathrm{N}_{\mathrm{R}} \times \mathrm{N}_{\mathrm{T}}$ optical MIMO antenna systems and is assumed that the channels are correlated in general and halfduplex which implies that the end-to-end transmission takes place in two distinct time slots.

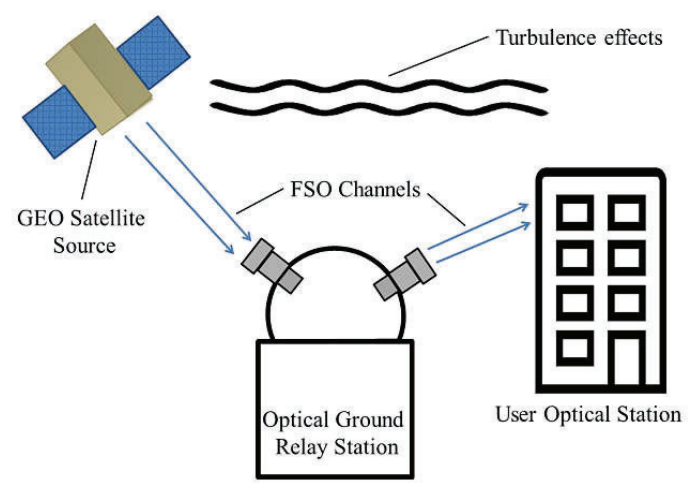

Figure 1. Network architecture. The GEO source, the OGRS gateway and the OGUS form optical MIMO systems over correlated, turbulent channels.

The channel spatial correlation depends on many factors i.e., the aperture size, the optical link distance, the type of turbulence and primarily the transmitter/receiver element spacing [16]-[17]. The spatial correlation coefficient between channels $n$ and $m$ of distance $d_{n m}$ is given by the following expression [16]-[17]:

$$
\rho_{n m}\left(d_{n m}\right)=\frac{R_{n m}\left(d_{n m}\right)}{\sqrt{\sigma_{I, n}^{2} \sigma_{I, m}^{2}}}
$$

where $-1 \leq \rho_{n m} \leq 1$ is the spatial correlation coefficient, $R_{n m}$ is the spatial covariance and $\sigma_{I}^{2}$ is the scintillation index which is defined as the normalized variance of the received irradiance. The spatial covariance of channels $n$ and $m$ is in turn [17]:

$$
R_{n m}\left(d_{n m}\right)=\frac{\left\langle I_{n} I_{m}\right\rangle}{\left\langle I_{n}\right\rangle\left\langle I_{m}\right\rangle}-1
$$

and the associated scintillation index of channel $n$ is [1], [7]: 


$$
\sigma_{I, n}^{2}=\frac{\left\langle I_{n}^{2}\right\rangle}{\left\langle I_{n}\right\rangle^{2}}-1
$$

where $\left\langle I_{n}\right\rangle$ is the average value of the received irradiance $I_{n}\left(\mathrm{~W} / \mathrm{m}^{2}\right)$. An analytical formula for the derivation of $R_{n m}$ is given in [16]-[17].

Fried's parameter $r_{c}(m)$ determines the channel correlation length and is another useful metric to quickly evaluate the possible correlation between optical channels [1], [3]:

$$
r_{c}=\left[0.42 k^{2} \sec (\zeta) \int_{A_{O G R S}}^{A_{G E O}} \mathrm{C}_{\mathrm{n}}^{2}(z) \mathrm{d} z\right]^{-3 / 5}
$$

Where $k=2 \pi / \lambda(\mathrm{rad} / \mathrm{m})$ is the wavenumber, $\lambda(\mathrm{m})$ is the communication wavelength, $\zeta$ is the zenith angle, $C_{n}^{2}(z)$ is the refractive index structure parameter, $z(m)$ is the altitude from ground and $A_{O G R S}, A_{G E O}$ are the altitudes of OGRS and GEO satellite. If the telescopes' spatial spacing is greater than $r_{c}$ then the channels can be assumed uncorrelated.

Regarding the communication technical characteristics, the simple On-Off-Keying NRZ (OOK-NRZ) modulation scheme is employed for transmission and the Direct Detection (DD) decoding scheme for reception while the optical wavelength is assumed equal to $\lambda=1550 \mathrm{~nm}$. The optical link pointing/tracking errors are assumed arbitrarily small and therefore neglected while a feedback channel provides CSIT knowledge to both source and relay. Finally, cloud-free conditions are presumed hence solely the atmospheric attenuation and turbulence are taken into account.

\section{THE PROPOSED METHODOLOGY}

\subsection{Channel modeling and important metrics}

The GEO-OGRS and OGRS-OGUS optical MIMO channel matrices will be referred to as $\mathbf{H}_{1}$ and $\mathbf{H}_{2}$ respectively of dimensions $N_{\mathrm{R}}$ x $N_{\mathrm{T}}$. The systemic expressions are the following:

$$
\begin{gathered}
\mathbf{Y}_{1}=\mathbf{H}_{1} \mathbf{X}_{1}+\mathbf{n}_{1} \\
\mathbf{Y}_{2}=\mathbf{H}_{2} \mathbf{X}_{2}+\mathbf{n}_{2}
\end{gathered}
$$

where $\mathbf{Y}_{1,2}$ are the received signal vectors of OGRS and OGUS respectively, $\mathbf{X}_{1,2}$ are the transmitted signal vectors of GEO and OGRS respectively and $\mathbf{n}_{1,2}$ are the optical detection noise vectors. There are three independent detection noise sources: The electronics noise e.g., thermal noise, the shot noise due to photonic interactions and the beat noise e.g., background radiation, amplified spontaneous emissions [1], [3]. The optical noise variance shall be assumed constant in this analysis although it's a function of the incident optical power. Therefore:

$$
\sigma_{n}^{2}=\sigma_{\text {elec }}^{2}+\sigma_{\text {shot }}^{2}+\sigma_{\text {beat }}^{2}=\text { const. }
$$

The optical signal propagation through the atmosphere is deteriorated by the atmospheric attenuation and turbulence that cause irradiance losses and scintillation [18]-[19]:

$$
I=I_{a v g} \cdot e^{2 X_{t}}
$$

where $I_{a v g}$ is the average received irradiance and $X_{t}$ is a random process representing the log-amplitude of the field fluctuations. The average irradiance term for a collimated Gaussian beam is [18]-[19]:

$$
I_{\text {avg }}=\frac{2}{\pi} P_{t} \cdot \eta_{t} \cdot \eta_{r} \cdot \eta_{a t m} \cdot 1 / W^{2}(D) \cdot \exp \left(-2 r^{2} / W^{2}(D)\right)
$$


where $\eta_{t}, \eta_{r}$ are the quantum efficiencies of the transmitter and receiver, $\eta_{a t m}$ is the atmospheric transmittance, $D(\mathrm{~m})$ is the optical link distance, $r$ is the radial distance from the beam center and $W(D)(\mathrm{m})$ is the beam waist after propagation of distance $D$. For perfect link pointing the $r=0$ and only the beam spreading effects take place.

The variance of $X_{t}$ is directly related to the scintillation index (SI) according to the following formula [1], [7]:

$$
\sigma_{I}^{2}=e^{4 \sigma_{X_{t}}^{2}}-1
$$

The special case of weak turbulence $(\mathrm{SI}<1)$ was thoroughly studied by Rytov from whom a theoretical closed-form expression for SI was derived [1], [7]:

$$
\sigma_{I}^{2}=2.25 k^{\frac{7}{6}} \sec ^{\frac{11}{6}}(\zeta) \int_{A_{O G R S}}^{A_{G E O}} \mathrm{C}_{\mathrm{n}}^{2}(z)\left(z-A_{O G R S}\right)^{\frac{5}{6}} d z
$$

Moreover, for satellite links with elevation angle greater than 20 degrees, $I$ is a log-normally distributed process with Probability Density Function (PDF) [1], [18]-[19]:

$$
\mathrm{f}_{I}(I)=\frac{1}{I \sqrt{2 \pi} \sigma_{I}} \exp \left\{-\frac{\left[\ln \left(I / I_{\text {avg }}\right)+\frac{1}{2} \sigma_{I}^{2}\right]^{2}}{2 \sigma_{I}^{2}}\right\}
$$

The channel capacity of the optical MIMO DF dual hop system in (bps/Hz) is derived from the Shannon theorem as [9][10]:

$$
C_{s}=\min \left(C_{1}, C_{2}\right)
$$

where the correspondent capacities in (13) are expressed as [10]-[11]:

$$
C_{1}=\frac{1}{2} \log _{2}\left|\mathbf{I}+\frac{1}{\sigma_{n}^{2}} \mathbf{H}_{1} \mathbf{Q}_{1} \mathbf{H}_{1}^{\mathbf{H}}\right|, C_{2}=\frac{1}{2} \log _{2}\left|\mathbf{I}+\frac{1}{\sigma_{n}^{2}} \mathbf{H}_{2} \mathbf{Q}_{2} \mathbf{H}_{2}^{\mathbf{H}}\right|
$$

where $\mathbf{I}\left(N_{\mathrm{R}} \mathrm{X} N_{\mathrm{R}}\right)$ is the identity matrix, $\mathbf{Q}_{\mathbf{1}}$ and $\mathbf{Q}_{2}$ are the covariance matrices of transmitted symbols $\mathbf{X}_{\mathbf{1}}$ and $\mathbf{X}_{\mathbf{2}}$ accordingly. From the Singular Value Decomposition (SVD) technique we obtain parallel, independent fading channels [10]:

$$
\begin{gathered}
\mathbf{H}_{\mathbf{1}}=\mathbf{U}_{1} \boldsymbol{\Lambda}_{1} \mathbf{V}_{1}{ }^{H}, \mathbf{H}_{2}=\mathbf{U}_{2} \boldsymbol{\Lambda}_{2} \mathbf{V}_{2}{ }^{H} \\
\mathbf{X}_{\mathbf{1}}=\mathbf{V}_{1} \widetilde{\mathbf{X}_{1}}, \mathbf{X}_{2}=\mathbf{V}_{2} \widetilde{\mathbf{X}_{2}}, \widetilde{\mathbf{Y}_{1}}=\mathbf{U}_{1}{ }^{H} \mathbf{Y}_{1}, \widetilde{\mathbf{Y}_{2}}=\mathbf{U}_{2}{ }^{H} \mathbf{Y}_{2} \\
\widetilde{\mathbf{Y}_{1}}=\boldsymbol{\Lambda}_{1} \widetilde{\mathbf{X}_{\mathbf{1}}}+\widetilde{\mathbf{n}_{1}}, \widetilde{\mathbf{Y}_{2}}=\boldsymbol{\Lambda}_{2} \widetilde{\mathbf{X}_{2}}+\widetilde{\mathbf{n}_{2}}
\end{gathered}
$$

The (14) are then transformed to:

$$
C_{1}=\frac{1}{2} \sum_{k=1}^{\operatorname{rank}\left(\mathbf{H}_{1}\right)} \log _{2}\left(1+\frac{P_{1, k} \cdot \gamma_{1, k}^{2}}{\sigma_{n}^{2}}\right), C_{2}=\frac{1}{2} \sum_{k=1}^{\operatorname{rank}\left(\mathbf{H}_{2}\right)} \log _{2}\left(1+\frac{P_{2, k} \cdot \gamma_{2, k}^{2}}{\sigma_{n}^{2}}\right)
$$

where $P_{i, k}(\mathrm{i}=1,2)$ are the transmitted powers regarding the GEO and OGRS transmitters, $\gamma_{i, k}(\mathrm{i}=1,2)$ are the eigenvalues of matrices $\boldsymbol{\Lambda}_{\mathbf{1}}$ and $\boldsymbol{\Lambda}_{\mathbf{2}}$.

\subsection{Problem formulation and methodology}

In this Section, the power allocation problem for an optical MIMO DF dual hop network is firstly structured and then a methodology is proposed that maximizes the system's capacity with respect to separate power constraints. The optimization problem is formulated as follows: 


$$
\begin{array}{ll}
\text { PA1: } & \left\{P_{1, k}^{*}, P_{2, k}^{*}\right\}=\underset{P_{i, k}}{\arg \max \min }\left(C_{1}, C_{2}\right) \\
\text { s.t. } & 0 \leq P_{1, k} \leq P_{\text {peak }, 1}, 0 \leq P_{2, k} \leq P_{\text {peak }, 2} \\
& \sum_{k=1}^{N_{T}} P_{1, k} \leq P_{\text {total }, 1}, \sum_{k=1}^{N_{T}} P_{2, k} \leq P_{\text {total }, 2}
\end{array}
$$

where $P_{1, k}^{*}, P_{2, k}^{*}$ are the optimum transmission powers of channel $k$ for GEO and OGRS accordingly, $P_{\text {peak }, i}(\mathrm{i}=1,2)$ is the peak transmitting power constraint and $P_{\text {total }, i}(\mathrm{i}=1,2)$ is the total transmitting power constraint. In order to solve the maxmin problem efficiently, the following transformation is applied:

$$
\begin{aligned}
& \text { PA2: }\left\{P_{1, k}^{*}, P_{2, k}^{*}\right\}=\underset{P_{i, k}}{\arg \max } \theta \\
& \text { s.t. } \theta \leq C_{1}, \theta \leq C_{2} \text { and }(20)
\end{aligned}
$$

where the slack variable $\theta$ is the minimum of $C_{1}, C_{2}$.

The PA2 is now in standard form to be solved with convex techniques since the objective function is the minimum of two concave functions and the constraints are also convex. Firstly, the Lagrangian is formed for the channel $k$ but we will omit the $k$ index notation [13]-[14]:

$L\left(P_{1}^{*}, P_{2}^{*}\right)=\theta-\lambda_{1}\left(\theta-C_{1}\right)-\lambda_{2}\left(\theta-C_{2}\right)-\mu_{1}\left(P_{1}^{*}-P_{p e a k, 1}\right)-\mu_{2}\left(P_{2}^{*}-P_{p e a k, ~}, 2\right)-v_{1}\left(\sum_{k=1}^{N_{T}} P_{1}^{*}-P_{\text {total }, 1}\right)-v_{2}\left(\sum_{k=1}^{N_{T}} P_{2}^{*}-P_{\text {total }, 2}\right)$

where $\lambda_{i}, \mu_{i}, v_{i}(\mathrm{i}=1,2)$ are the non-negative Lagrange multipliers. Then the Karush-Kuhn-Tucker conditions are necessary and sufficient therefore we obtain [13]-[14]:

$$
\nabla \cdot L\left(P_{1}^{*}, P_{2}^{*}\right)=0 \Rightarrow\left\{\begin{array}{l}
\frac{\partial L\left(P_{1}^{*}, P_{2}^{*}\right)}{\partial P_{1}^{*}}=0 \\
\frac{\partial L\left(P_{1}^{*}, P_{2}^{*}\right)}{\partial P_{2}^{*}}=0
\end{array}\right.
$$

By calculating the first derivatives and applying the complementary slackness conditions we get:

$$
P_{1}^{*}=\min \left\{P_{\text {peak }, 1}, \max \left\{\frac{1}{v_{1}}-\frac{\sigma_{n}^{2}}{\gamma_{1}^{2}}, 0\right\}\right\}, P_{2}^{*}=\min \left\{P_{\text {peak }, 2}, \max \left\{\frac{1}{v_{2}}-\frac{\sigma_{n}^{2}}{\gamma_{2}^{2}}, 0\right\}\right\}
$$

where the dual multipliers $v_{1}, v_{2}$ are computed numerically from the total power equations given below:

$$
\sum_{k=1}^{N_{T}} P_{1, k}^{*}=P_{\text {total }, 1}, \sum_{k=1}^{N_{T}} P_{2, k}^{*}=P_{\text {total }, 2}
$$

A root-finding algorithm such as the iterative subgradient method or the bisection method can be equally employed to solve for the $v_{1}, v_{2}$ optimal values. It is shown from (27) that $v_{i}$ lie in the interval $\left(0,1 / P_{\text {peak }, i}\right)$ so an initial choice e.g. $v_{i}(0)=0.5 / P_{p e a k, i}$ and a small fixed step size e.g. 1e-3 are usually sufficient to rapidly converge to the optimum solution. 


$$
\sum_{k=1}^{N_{T}}\left(\frac{1}{v_{i}}-\frac{\sigma_{n}^{2}}{\gamma_{i}^{2}}\right)=P_{\text {total }, i} \Rightarrow \frac{1}{v_{i}}=\frac{1}{N_{T}}\left[P_{\text {total }, i}+\sum_{k=1}^{N_{T}} \frac{\sigma_{n}^{2}}{\gamma_{i}^{2}}\right] \Rightarrow \frac{1}{v_{i}} \geq \frac{P_{\text {total }, i}}{N_{T}} \geq P_{\text {peak }, i} \Rightarrow v_{i} \leq \frac{1}{P_{\text {peak }, i}}
$$

\section{NUMERICAL RESULTS}

In this Section, the proposed methodology is evaluated through numerical simulations for various network topologies and settings and its performance is tested against other known power allocation methods. Specifically, the impact of spatial correlation on the system's capacity is examined as well as the sum rate difference between the proposed allocation scheme and the Even Power Allocation algorithm. Finally, the special case of poor GEO-OGRS channel conditions is considered to investigate the power efficiency of the proposed scheme.

To begin with, the case of a $3 \times 3$ optical MIMO is assumed for both GEO-OGRS, OGRS-OGUS links under atmospheric attenuation and weak scintillation conditions. In order to solely examine the impact of correlation, the average irradiance of the channels is normalized to unity while the scintillation index is calculated using the following input parameters: $\lambda=1550 \mathrm{~nm}, \zeta=30^{\circ}, \mathrm{A}_{\mathrm{OGRS}}=2000 \mathrm{~m}, \mathrm{~A}_{\mathrm{GEO}}=35786 \mathrm{~km}$ while the Hufnagel-Valley model was employed for the $C^{2}{ }_{\mathrm{n}}$ [1], [7]. Furthermore, we assume correlated optical channels on the transmitter side for the GEO-OGRS links i.e., the columns of $\mathbf{H}_{1}$ are correlated, and on the receiver side for the OGRS-OGUS links i.e., the rows of $\mathbf{H}_{2}$ are correlated. As an example, the correlation matrix of first row of $\mathbf{H}_{1}$ is given below:

$$
\mathbf{H}_{1}=\left[\begin{array}{lll}
h_{11} & h_{12} & h_{13} \\
h_{21} & h_{22} & h_{23} \\
h_{31} & h_{32} & h_{33}
\end{array}\right] \stackrel{\text { Correalted }}{\underset{\text { Columns }}{\longrightarrow}} \operatorname{Corr}\left(\mathbf{H}_{1}\right)=h_{12} h_{11}^{*}\left[\begin{array}{cccc}
1 & \rho & \rho \\
\rho^{*} & 1 & \rho \\
h_{13} & \rho^{*} & \rho^{*} & 1
\end{array}\right]
$$

For the first simulation, the peak transmitting power is fixed at $P_{\text {peak }, 1}=P_{\text {peak }, 2}=1 \mathrm{~W}$ and the system capacity is computed using the proposed methodology for a range of total available powers $P_{\text {total }, 1}=P_{\text {total }, 2}=P_{\text {total }}$ from $1 \mathrm{~W}$ to $3 \mathrm{~W}$ and for $\rho=0,0.5,0.95$. The optical channels are subjected to weak scintillation thus are log-normally distributed and 1000 channel realizations were generated for each simulation run. In Figure 2, the overall system capacity is exhibited as a function of GEO's and OGRS's total power for different channel correlation coefficients.

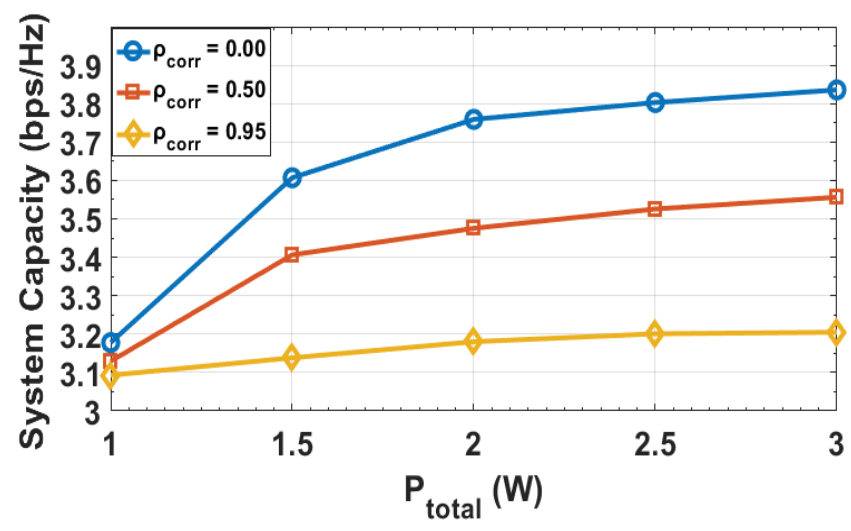

Figure 2. System capacity versus the total available power for various channel correlation coefficients. The higher the correlation the lower the network's spectral efficiency.

It is observed that the system capacity increases with the increment of total power. However, the correlated channels are significantly worse for data transmission than the uncorrelated ones since the capacity falls off with higher values of the correlation coefficient. Moreover, as the $\rho_{\text {corr }} \rightarrow 1$ the system capacity becomes less sensitive to the total available power by only showing a slight increase with larger total power. 
Regarding the second simulation, the peak transmitting power is again fixed at $P_{\text {peak }, 1}=P_{\text {peak }, 2}=1 \mathrm{~W}$ and the proposed methodology shall be juxtaposed with the Even Power Allocation (EPA) Algorithm for a range of total available powers $P_{\text {total }, 1}=P_{\text {total }, 2}=P_{\text {total }}$ from $1 \mathrm{~W}$ to $3 \mathrm{~W}$. The EPA algorithm is basically a reference algorithm in order to demonstrate the superiority of the proposed power allocation methodology in terms of bit rate-based performance.

In Figure 3, the performance comparison is depicted. It is clear that the proposed methodology outperforms the EPA algorithm by far, reaching greater data rates. Nevertheless, at $3 \mathrm{~W}$ the proposed methodology works similarly to the EPA.

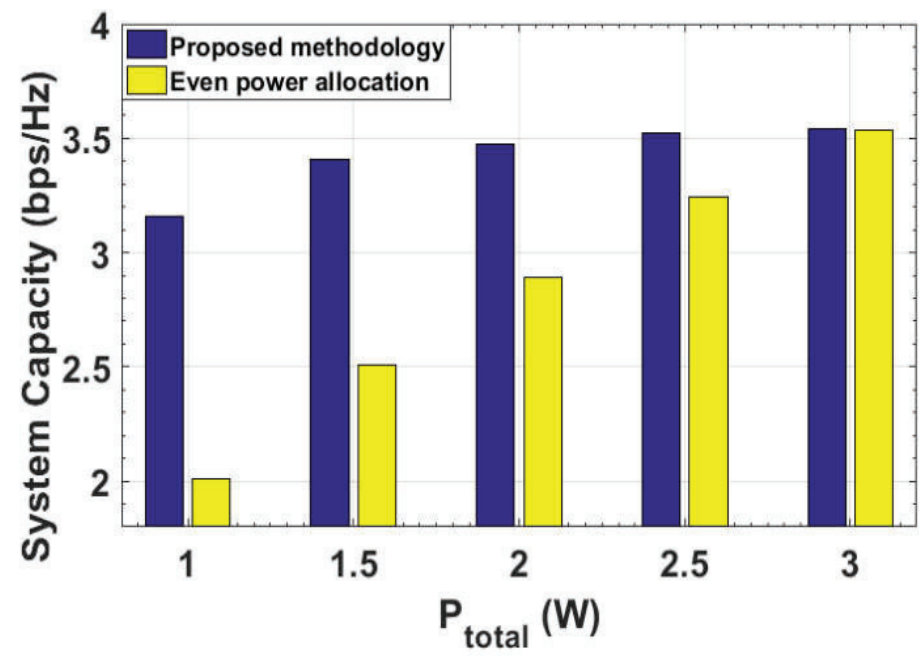

Figure 3. Performance comparison between the proposed power allocation methodology and the even power allocation algorithm. The proposed methodology achieves higher data rates over all total power values and outperforms the simpler even allocation. Then, at $3 \mathrm{~W}$ the two algorithms perform the same as expected.

For the last simulation, it is assumed that the channel conditions of GEO-OGRS links are of poor quality (high attenuation, scintillation, correlation) and so the GEO transmits with full total available power $P_{\text {total, } 1}=3 \mathrm{~W}$. The proposed methodology is then employed over a range of OGRS's $P_{\text {total, } 2}$ from $1 \mathrm{~W}$ to $3 \mathrm{~W}$ and the two sub-system capacities are computed. In Figure 4 the two channel capacities are exhibited to show that in this case, the OGRS can utilize $1.25 \mathrm{~W}$ total power to obtain the network's maximum power efficiency.

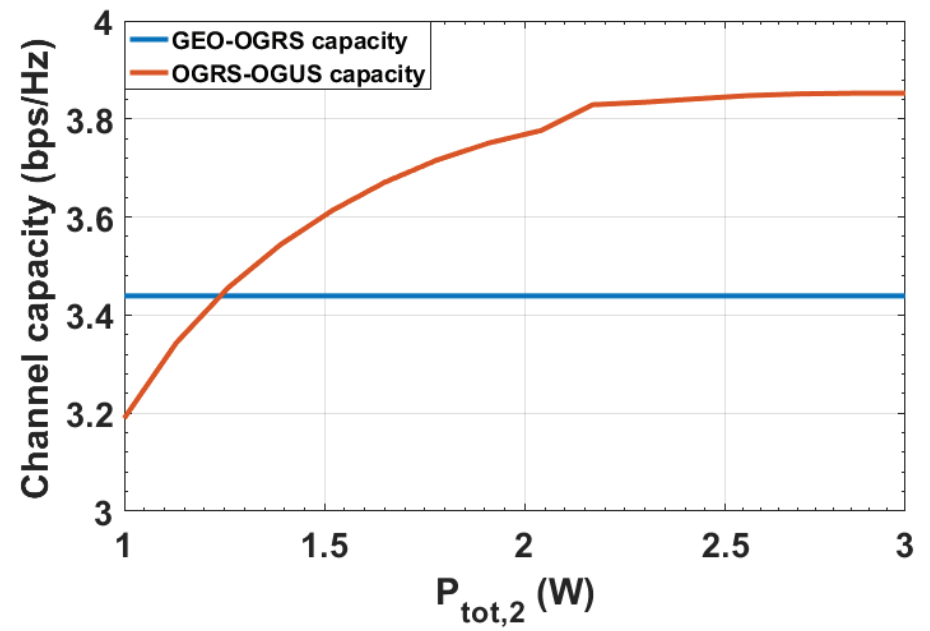

Figure 4. Channel capacities of GEO-OGRS, OGRS-OGUS in poor source-relay conditions. The $P_{\text {total,1 }}=3 \mathrm{~W}$ is fixed while the $P_{\text {total, } 2}$ is sweeped from $1 \mathrm{~W}$ to $3 \mathrm{~W}$ using the proposed methodology. The OGRS needs no more than $1.25 \mathrm{~W}$ to reach the maximum capacity since the network capacity is the minimum of them two. 


\section{CONCLUSION}

In this paper hybrid optical satellite links are investigated in a MIMO DF dual hop network formation. A power allocation methodology is then presented and proposed for the optimization of the system's capacity under separate peak and total available power constraints. The power allocation problem is initially structured as a convex optimization problem and then solved with the corresponding convexity theory and SVD method whilst taking into account the spatial channel correlation, the atmospheric attenuation and the turbulence strength. Finally, numerical simulations are executed to examine the impact of correlation on system capacity, to evaluate the proposed algorithm's performance through comparison with the EPA algorithm and to give insight to the special case of poor source-relay conditions regarding the system's power efficiency. The derived numerical results are presented and remarks are made validating the proposed methodology in various network topologies and settings.

\section{REFERENCES}

[1] H. Hemmati, "Near-Earth Laser Communications," Boca Raton: CRC Press, (2009).

[2] H. Kaushal, G. Kaddoum, "Optical Communication in Space: Challenges and Mitigation Techniques," in IEEE Comm. Surv. Tutor., 19, 57-96 (2016).

[3] Kapsis, T. T., \& Panagopoulos, A. D., "Optimum Power Allocation Based on Channel Conditions in Optical Satellite Downlinks," Wireless Personal Communications, 1-17 (2020).

[4] N. K. Lyras, C. N. Efrem, C. I. Kourogiorgas, A. D. Panagopoulos and P.D. Arapoglou, "Optimizing the Ground Network of Optical MEO Satellite Communication Systems," in IEEE Systems Journal, 14(3), 39683976 (2020).

[5] C. N. Efrem, N. K. Lyras, C. I. Kourogiorgas, A. D. Panagopoulos and P. Arapoglou, "Cloud Free LOS Probability Estimation for MEO Optical Satellite Systems and Optical Satellite Network Dimensioning," 14th European Conference on Antennas and Propagation (EuCAP), 1-5 (2020).

[6] N.K. Lyras, C.I. Kourogiorgas, A.D. Panagopoulos, "Cloud Attenuation Statistics Prediction from Ka-Band to Optical Frequencies: Integrated Liquid Water Content Field Synthesizer," in IEEE Trans. Antennas Propag., 65, 319-328 (2017).

[7] L. Andrews, R. Phillips, "Laser Beam Propagation through Random Media," 2nd ed., SPIE Press: Bellingham, WA,USA, (2005).

[8] Hassan, N., \& Fernando, X., "Massive MIMO wireless networks: An overview," Electronics, 6(3), 63 (2017).

[9] Fitzek, F. H., \& Katz, M. D., "Cooperation in wireless networks: principles and applications," New York: Springer (2006).

[10]Lu, F., Liu, C., Fu, Y., \& Zhao, H. A., "Joint Power Allocation for DF MIMO Relay Links with Hybrid Power Constraints," in IEEE 8th International Conference on Wireless Communications, Networking and Mobile Computing,1-4 (2012).

[11] Hammerstrom, I., \& Wittneben, A., "Power allocation schemes for amplify-and-forward MIMO-OFDM relay links," in IEEE Transactions on Wireless Communications, 6(8), 2798-2802 (2007).

[12]Dabiri, M. T., \& Sadough, S. M. S., "Performance analysis of all-optical amplify and forward relaying over lognormal FSO channels," Journal of Optical Communications and Networking, 10(2), 79-89 (2018).

[13] S. Boyd, L. Vandenberghe, "Convex Optimization," Univ. Press: Cambridge, U.K., (2004).

[14]D. P. Bertsekas, "Constrained Optimization and Lagrange Multiplier Methods," New York: Academic Press, (1982).

[15]G. Scutari, D. P. Palomar and S. Barbarossa, "The MIMO Iterative Waterfilling Algorithm," in IEEE Transactions on Signal Processing, 57(5), 1917-1935 (2009).

[16] T. V. Pham, T. C. Thang and A. T. Pham, "Average achievable rate of spatial diversity MIMO-FSO over correlated Gamma-Gamma fading channels," IEEE/OSA Journal of Optical Communications and Networking, 10(8), 662-674 (2018).

[17] Chen, Z., Yu, S., Wang, T., Wu, G., Wang, S., \& Gu, W., "Channel correlation in aperture receiver diversity systems for free-space optical communication," Journal of Optics, 14(12), 125710 (2012).

[18] T. T. Kapsis, N. K. Lyras, and A. D. Panagopoulos, "Long Term Irradiance Statistics for Optical GEO Downlinks: Validation with ARTEMIS Experimental Measurements," in Prog. Electrom. Res. Letters, 82, 8994, (2019). 
[19] T. T. Kapsis, N. K. Lyras, C.I. Kourogiorgas and A. D. Panagopoulos, “Time Series Irradiance Synthesizer for Optical GEO Satellite Downlinks in 5G Networks,” in Future Internet, 11(6), 131 (2019). 